\title{
From distances to galaxy evolution and the dark matter problem
}

\author{
F. Combes
}

\author{
Observatoire de Paris, LERMA, 61 Av. de l'Observatoire, 75014 Paris, France \\ e-mail: francoise.combes@obspm.fr
}

Thirty-two years ago, Brent Tully and Richard Fisher wrote this ground-breaking article on a method of determining galaxy distances that is independent of redshift. This method is now the most widely used one for spiral galaxies. They proposed using a correlation between a distance-independent observable for spiral galaxies, the global neutral atomic hydrogen (HI) profile width, and their absolute magnitude or global luminosity, to indicate distances. It was not the first time that comparable correlations were used as distance indicators (e.g. Roberts 1962; Balkowski et al. 1973; Shostak 1974), and the virial theorem was invoked as the basis. But the simple correlation between luminosity and rotational velocity had not yet been approached. Also many other correlations had been studied, such as a correlation with morphological type, so that the breakthrough in this paper was to show that the correlation with type was secondary (since early type objects are more massive and luminous) and that the principal and original correlation was with luminosity.

As Brent Tully recalls now: "Opik in the 1920's used the virial theorem to get a distance to M31, measuring luminosity, radius and rotation rate and assuming a reasonable stellar value for M/L. He got a distance to M 31 that was earlier and more accurate than determined by Hubble using cepheids.

Then several groups in the 1960-70's used a variation of the virial theorem as a distance indicator: they assumed $L \propto r V^{2}$ with a constant determined from observations. This was one of the many poor ways to get distances in those days. Notice that there is distance dependence in 2 of the 3 parameters, and the $r V^{2}$ dependence is forced.

The relation proposed by Fisher and myself is $L \propto V^{\alpha}$ so only one of two parameters are dependent on distance and the power law is to be discovered. We already knew those things at the time we wrote the paper, but we couldn't have anticipated the tightness of the correlation".

The difficulty of convincing the community of the quality of the correlation was to reduce the errors that flawed early attempts to calibrate it: by more accurately knowing the distance of nearby galaxies, their inclination corrections of the intrinsic rotational velocity, and corrections to magnitude. Tully and Fisher succeeded in obtaining good calibrators with careful corrections, and then applied the relation to finding the distance to the Virgo and Ursa Major cluster. They also derived an estimate of the Hubble constant, $H_{0}=80 \mathrm{~km} \mathrm{~s}^{-1} \mathrm{Mpc}^{-1}$, not so far from the accepted value today (Sakai et al. 2000).

The distance to Virgo that they determined (13.2 Mpc) was inconsistent with previous determinations of $19.5 \mathrm{Mpc}$
(Sandage \& Tammann 1974), and they argued that the reason was the previous inclusion of the Virgo II southern extension, which does not belong to the same cluster. An interesting feature of their work is that they proposed both the correlation of the HI line width with the magnitude and the diameter of spiral galaxies. Given the uncertainties and the difficulty defining diameters precisely, only the total luminosity was retained in later literature.

Although their correlation could also help for understanding galactic structure, Tully \& Fisher (1977) put the emphasis on discovering a new method of determining distances, in competition with other distance indicators used as standard candles (peculiar stars or giant HII regions). The correlation turned out to be an impressive tool for distinguishing peculiar motions from expansion and mapping the large-scale structure of the local universe (Dekel 1994). At the beginning, most citations of the 1977 paper were coming from individual galaxy studies, then, and more and more from the large-scale structures and the expansion rate of super-clusters. It was soon realized that the red or infrared magnitudes were much better correlated to the HI line width than the visible one (Aaronson et al. 1979; Giovanelli et al. 1997) and that extinction was an important source of scatter. The infrared luminosity/H I velocity-width relation was a power law with slope 4 . Very soon the method was called the Tully-Fisher relation (TFR), parallel to the Faber-Jackson (1976) equivalent relation for elliptical galaxies. There were many attempts to find a second parameter able to reduce the scatter, such as the morphological type (Rubin et al. 1980); however, none actually succeeded, and the TFR remains a unique relation on one dimension (but not a plane).

The TFR has revealed itself to be extremely rich in applications to the origin of galaxies, and even the scatter in the relation was interpreted physically. It could help for understanding galaxy evolution along the Hubble sequence, bars in galaxies, low-surface-brightness objects, or luminosity evolution (Burstein 1982; Sprayberry et al. 1995; Zwaan et al. 1995; Barton et al. 2001; Courteau et al. 2003). This type of work from scaling physical relations was developed in parallel for elliptical galaxies, which are more difficult to deproject, and deep development beyond the Faber-Jackson relation were worked out towards the fundamental plane (Djorgovski et al. 1987). Terlevich et al. (1981) discovered that a second parameter was needed to determine the position of any elliptical galaxy, in contrast to spiral galaxies. 
One part of the TFR may be explained by the virial relation, which must be satisfied in galaxies, i.e., that the velocity width (or peak of the rotation curve, $V$ ) has to vary as the square root of the total mass $M$, divided by the characteristic radius $R$. Since the TFR involves the luminosity, which is proportional to the mass of baryons $M_{\mathrm{b}}$, we write the virial relation as $k M_{\mathrm{b}}=V^{2} R$, where $k$ is proportional to the total-to-baryon mass ratio. But then another relation is required to account for the TFR, and this is typically a scaling relation between the baryon mass (or luminosity) and the size. For a large category of high surface brightness galaxies (HSB), such a relation was found by Freeman (1970) as a constant surface density $\mu_{\mathrm{HSB}}$, since $M_{b}=\mu_{\mathrm{HSB}} \pi R^{2}$. For all HSB galaxies, the combination of this relation and the virial theorem yields $\mu_{\mathrm{HSB}} \pi k^{2} M_{\mathrm{b}}=V^{4}$, and the TFR can be understood as a constant baryon-to-total mass ratio. When another category of galaxies of low surface brightness (LSB) were discovered, violating Freeman's relation (Sprayberry et al. 1995), it was found that they also satisfied the same TFR, i.e. $\mu_{\mathrm{LSB}} \pi k_{\mathrm{LSB}}^{2} M_{\mathrm{b}}=V^{4}$, or in other words that $\mu k^{2}$ was an invariant for all categories. Indeed LSB proportionally have a larger fraction of dark matter than HSB, and their low values of $\mu$ are compensated for by a high $k$, although they still have a low surface density, as far as total mass is concerned (low $\mu k$ ), as confirmed by their slowly rising rotation curves.

The baryonic mass is usually used here, deduced from the luminosity through a well-motivated dependence of the stellar mass-to-light ratio on the mass. The scaling law is fundamental to a better understanding of the processes of galaxy formation (e.g. Courteau et al. 2007) and has been continuously used for many peculiar classes of galaxies, unlike the normal ones (e.g. Iodice et al. 2003), or as a function of environment (Biviano et al. 1990).

The TFR is one of the fundamental features sought in the evolution of galaxies as a function of redshift. Many studies try to establish how the relation is maintained or retrieved, or it varies (Böhm et al. 2004; Conselice et al. 2005). The results of observations remain preliminary, but in the future, the comparison of the TFR at high $z$ with the predictions from the simulations will certainly be one of the major tools for understanding galaxy formation and evolution.

Last but not least, the TFR is fundamental to better understanding the behavior of the dark matter in spiral galaxies. Since on the one hand, the magnitude (or luminosity) accounts for the visible baryons and, on the other, the rotational velocity traces the gravitational potential provided by dark matter, the relation is an index of the strong link between baryonic and dark matter. The standard model of CDM has difficulty reproducing the formation of galaxy in particular and its scaling laws, either through fully numerical (Gnedin et al. 2007) or semi-analytical simulations (Somerville \& Primack 1999). While the slope of the TFR is easy to retrieve, the zero point is a crucial test of the angular momentum problem in disk formation (Steinmetz \& Navarro 1999). The TFR can be naturally explained in hierarchical models, but its normalization and evolution depend strongly on the star formation algorithm chosen and on the cosmological parameters that determine the universal baryon fraction and the assembly time of galaxies with different masses.
Since the TFR traces a strong link between the dark matter and baryonic content of galaxies, it was interesting to see a break for dwarf Irr galaxies, which are known to be dominated by dark matter: in the optical TFR, galaxies with rotational velocities lower than $90 \mathrm{~km} \mathrm{~s}^{-1}$ fall below the normal relation. These faint galaxies, however, are very rich in gas, and the gas does not contribute to the flux in the optical bands. It is sufficient to replace the optical luminosity by the total visible mass of the disk (gas and stars) to restore the relation, which is then called the baryonic TFR (McGaugh et al. 2000).

The TFR thus appears to be a fundamental relation between rotation velocity and total baryonic mass. It therefore comes as no surprise that the TFR has inspired cosmological models to solve the dark matter problem in terms of modified gravity (Milgrom 1983). In this modification of Newtonian gravity, a mass-rotation velocity relation of the form $M \propto V^{4}$ is automatically obtained.

The TFR is now an outstanding feature in galaxy physics, so widely acknowledged that it has entered current language and is used without even being cited. The citation rate of the original paper still increases year after year. This fundamental study aimed at providing a distance indicator has not only fullfilled its first goal beyond expectations, but also surpassed these first steps and extended them to new areas of large-scale structure, galaxy physics, models of dark matter, galaxy formation, and evolution.

\section{References}

Aaronson, M., Huchra, J., \& Mould, J. 1979, ApJ, 229, 1

Balkowski, C., Bottinelli, L., Chamaraux, P., et al. 1973, A\&A, 25, 319

Barton, E. J., Geller, M. J., Bromley, B. C., et al. 2001, AJ, 121, 625

Biviano, A., Giuricin, G., Mardirossian, F., \& Mezzetti, M. 1990, ApJS, 74, 325

Bohm, A., Ziegler, B. L., Saglia, R. P., et al. 2004, A\&A, 420, 97

Burstein, D. 1982, ApJ, 253, 539

Conselice, C. J., Bundy, K., Ellis, R. S., et al. 2005, ApJ, 628, 160

Courteau, S., Andersen, D. R., Bershady, M. A., et al. 2003, ApJ, 594, 208

Courteau, S., Dutton, A. A., van den Bosch, F. C., et al. 2007, ApJ, 671, 203

Dekel, A. 1994, ARA\&A, 32, 371

Djorgovski, S., \& Davis, M. 1987, ApJ, 313, 59

Faber, S. M., \& Jackson, R. E. 1976, ApJ, 204, 668

Freeman, K. C. 1970, ApJ, 160, 811

Giovanelli, R., Haynes, M. P., Herter, T., et al. 1997, AJ, 113, 22

Gnedin, O. Y., Weinberg, D. H., Pizagno, J., Prada, F., \& Rix, H.-W. 2007, ApJ, 671,1115

Iodice, E., Arnaboldi, M., Bournaud, F., et al. 2003, ApJ, 585, 730

McGaugh, S. S., Schombert, J. M., Bothun, G. D., \& de Blok, W. J. G. 2000, ApJ, 533, L99

Milgrom, M. 1983, ApJ, 270, 365

Roberts, M. S. 1962, AJ, 67, 431

Rubin, V. C., Burstein, D., \& Thonnard, N. 1980, ApJ, 242, L149

Sakai, S., Mould, J. R., Hughes, S., et al. 2000, ApJ, 529, 698

Sandage, A., \& Tammann, G. A. 1974, ApJ, 194, 559

Sanders, R. H., \& McGaugh, S. S. 2002, ARA\&A, 40, 263

Shostak, G. S. 1974, ApJ, 187, 19

Somerville, R. S., \& Primack, J. R. 1999, MNRAS, 310, 1087

Sprayberry, D., Bernstein, G. M., Impey, C. D., \& Bothun, G. D. 1995, ApJ, 438, 72

Steinmetz, M., \& Navarro, J. 1999, ApJ, 513, 555

Terlevich, R., Davies, R. L., Faber, S. M., \& Burstein, D. 1981, MNRAS, 196, 381

Tully, R. B., \& Fisher, J. R. 1977, A\&A, 54, 661

Zwaan, M. A., van der Hulst, J. M., de Blok, W. J. G., \& McGaugh, S. S. 1995, MNRAS, 273, L35 